\title{
Towards the Derivation of Stress Intensity Factors by Parametric Modelling of Full-Field Thermoelastic Data
}

\author{
R.I Hebb ${ }^{1, a}$, J. M. Dulieu-Barton ${ }^{1, b}$, K. Worden ${ }^{2, c}$, P. Tatum ${ }^{3,}$ \\ University of Southampton, School of Engineering Sciences, Highfield, Southampton, SO17 1BJ, \\ UK \\ *AWE, Aldermaston, Reading, Berkshire, RG7 4PR, UK \\ + Department of Mechanical Engineering, University of Sheffield, Mappin Street, \\ Sheffield, S1 3JD, UK \\ ${ }^{a}$ rih103@soton.ac.uk, ${ }^{b}$ janice@soton.ac.uk, ${ }^{\mathrm{c}}$ k.worden@sheffield.ac.uk
}

Keywords: thermoelastic stress analysis, stress intensity factors, thermoelasticity

\begin{abstract}
Thermoelastic Stress Analysis (TSA) is a well-established full-field technique for experimental stress analysis that has proved to be extremely effective for studying stress fields in the vicinity of cracks. Recently, work has focused on the observation that the stress-sum contours (isopachics) obtained from TSA take the form of a cardioid. Genetic Algorithms (GAs) and Differential Evolution (DE) have proved successful for accurate parameter estimation of the cardioids, thus allowing the SIFs to be calculated. Originally, some curve-fits indicated that a pure cardioid form is inappropriate for the base model, especially for mixed-mode cracks. The deviation from the cardioid form has been shown to be due to higher-order terms within the stress function. The objective of the current paper is to use a modified version of the original methodology (that fitted parameters to a single isopachic) to find the higher-order parameters from the entire data field obtained from the TSA.
\end{abstract}

\section{Introduction}

Effective diagnosis and prognosis algorithms for Non-Destructive Testing (NDT) and Structural Health Monitoring (SHM) require an in-depth understanding of stress/strain fields in the neighbourhood of cracks and damage. In Thermoelastic Stress Analysis (TSA) the observed isopachics (stress-sum contours) can be used to derive the Stress Intensity Factors (SIFs), which dictate the crack propagation and can be used to define the remnant life of the component. In previous work, by the authors, it was assumed that the isopachics took the form of cardioids. The approach was based on the first order Westergaard equations [1], or equivalently the singular terms in the eigenfunction expansion of Williams [2]. The effectiveness of using the cardioid form to derive the SIFs has already been demonstrated in [3, 4], where software and algorithms were developed that related the SIFs to the cardioid area and certain tangents to the curve.

Recent work [5] directly fitted the cardioid form to extracted isopachics to estimate the crack-tip SIFs. The fitting was performed using either a Genetic Algorithm (GA) [5] or Differential Evolution (DE) [6]. An added advantage of the approach is that the crack-tip is located in the data field, which can be difficult to do by visual inspection. However, some of the results indicated that a cardioid form was unsuitable for the base model. Each isopachic contour rotated by different amounts and produced a set of un-nested contours, which the first order Westergaard equations could not accommodate [7]. As a result the GA generated inaccurate values of the SIFs.

The object of the current paper is to incorporate the higher-order terms of the Williams expansion into the method and show that inclusion of these terms accommodates the rotation of the isopachics. It is demonstrated that inclusion of the higher-order terms is essential for accurate analysis. It was suggested in [7] that the omission of the higher-order terms in of the Williams expansion may have been responsible for the previous inability to explain the observed rotations and the work described 
in this paper clearly shows that this is the case. It has been necessary to process the entire thermoelastic data set rather than selecting individual isopachics hence the work described here significantly advances the data processing capabilities.

\section{Theory}

Thermoelastic stress analysis is based on the measurement of small temperature changes that occur in solids on the application of a cyclic stress. It can be shown that the temperature changes are proportional to the changes in the sum of principal stresses [8]. The temperature changes are measured using a highly sensitive infra-red detector. Modern infra-red detectors have been radiometrically calibrated and so are able to directly output a temperature measurement. For a homogeneous, isotropic material, the change in temperature can be related to the change in stress via:

$$
\Delta T=-K T \Delta\left(\sigma_{1}+\sigma_{2}\right)
$$

where $\Delta T$ is the measured change in temperature, $K$ is the thermoelastic constant for the material, $T$ is the absolute temperature of the specimen and $\Delta\left(\sigma_{1}+\sigma_{2}\right)$ is the change in principal stresses.

The original approach $[5,6]$, related the SIFs to the first order Westergaard equations. In the region surrounding the crack-tip (but not the immediate vicinity where plasticity is a contributing factor and Linear Elastic Fracture Mechanics (LEFM) does not apply) the stress sum is related to the mode I and II SIFs $\left(K_{I}\right.$ and $\left.K_{I I}\right)$ by:

$$
\Delta\left(\sigma_{1}+\sigma_{2}\right)=\Delta\left(\sigma_{x}+\sigma_{y}\right)=\frac{2 K_{I}}{\sqrt{2 \pi r}} \cos \left(\frac{\theta}{2}\right)-\frac{2 K_{I I}}{\sqrt{2 \pi r}} \sin \left(\frac{\theta}{2}\right)
$$

where $r$ and $\theta$ are polar coordinates with the origin at the crack-tip, and $\theta$ measured anti-clockwise from the crack-line.

Rearranging Eq. 2 for $r$ gives:

$$
r=\frac{K_{I}^{2}+K_{I I}^{2}}{\pi}\left(\frac{K T}{\Delta T}\right)^{2}\left[1+\cos \left(\theta+2 \varphi_{0}\right)\right]
$$

which is well-known polar representation of the cardioid curve and $\varphi_{0}=\tan ^{-1}\left(K_{I I} / K_{I}\right)$ is the rotation of the cardioid.

Eq. 3 shows that for the first order Westergaard case, curves of constant temperature change will form nested cardioids, all with the same rotation angle. Including the higher order terms, the Williams expansion leads to:

$$
\frac{\Delta T}{K T}=\frac{2 \sqrt{K_{I}^{2}+K_{I I}^{2}}}{\sqrt{2 \pi r}} \cos \left(\frac{\theta}{2}+\varphi_{0}\right)+T_{s}+2 \sqrt{A_{I}^{2}+A_{I I}^{2}} \sqrt{r} \cos \left(\frac{\theta}{2}-\varphi_{1}\right)
$$

where $A_{I}$ and $A_{I I}$ are geometry-dependent constants, $\varphi_{I}=\tan ^{-1}\left(A_{I I} / A_{I}\right)$ and $T_{s}$ is the T-stress. It is clear from Eq. 4 that the $O\left(r^{1 / 2}\right)$ term will cause a secondary rotation of the field of contours and will deform the cardioid. Physically, regions close to the crack-tip are dominated by the $O\left(r^{-1 / 2}\right)$ term with the isopachics having a rotation of $\varphi_{0}$, but the $O\left(r^{1 / 2}\right)$ term will start to dominate the field further from the tip, with a rotation of $\varphi_{1}$. This effect can clearly be seen in Fig. 1, where a contour plot for the lowest-order William's expansion is shown with the higher-order expansion

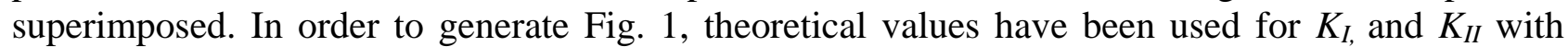
arbitrary values set for the other parameters. The crack tip is positioned at $45^{\circ}$ and is indicated. The 
contours generated when the higher-order terms are included clearly rotate towards the horizontal with increasing distance from the crack tip, showing, as expected, that the higher-order terms have more influence away from the crack-tip.

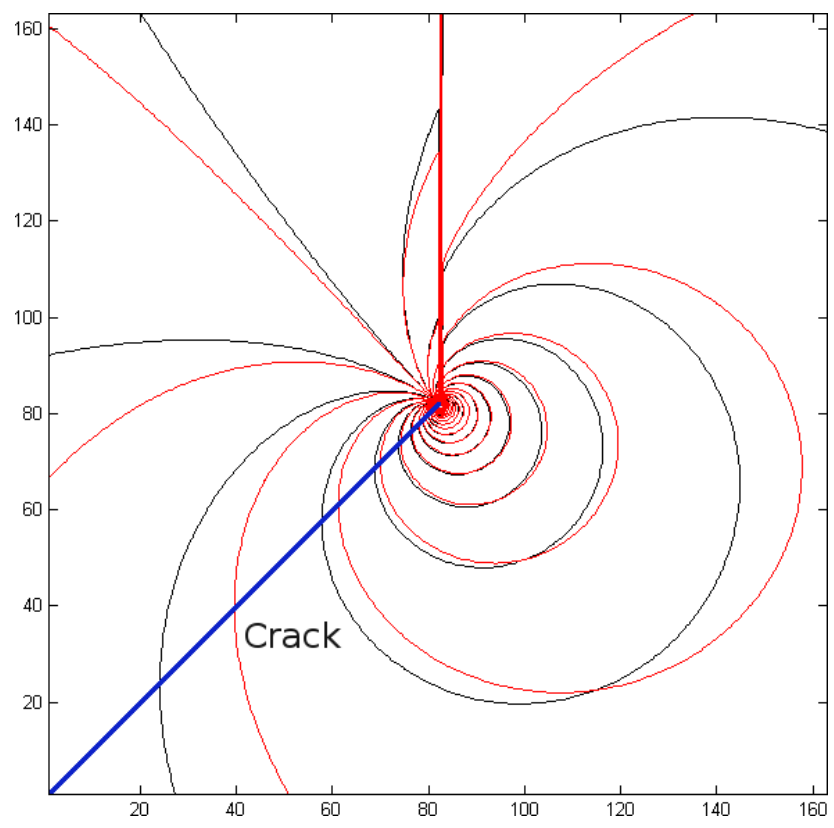

Figure 1: Contour plots of the lowest-order Williams expansion (black) and the Williams expansion including the higher-order terms (red)

\section{Test specimen and set-up}

The dimensions of the plate specimen used in this work are shown in Fig. 2. The material is Duralumin ${ }^{\circledR}$ and has dimensions $300 \times 300 \mathrm{~mm}$ with a thickness of $1.5 \mathrm{~mm}$. A slot at an angle of $\beta$ $=45^{\circ}$ and of total length $50 \mathrm{~mm}$ has been spark eroded from a centrally located hole (in this case the slot is used to simulate a real crack). 15 holes top and bottom are used to mount the plate into the loading jig. To strengthen the area around the holes when loaded, 4 Duralumin ${ }^{\circledR}$ tabs are bonded to both sides, top and bottom, to spread the clamping load. The specimen was mounted in an Instron 8800 servo-hydraulic test machine using knuckle joints to ensure that no bending moments are introduced into the system. Fig. 3 shows a schematic of the loading jigs. Preparation of the specimen for testing required the surface of the plate to be cleaned and two passes of matt black paint to be applied. The purpose of the paint is to reduce reflections from the plate, and to increase the emissivity of the specimen. The specimen was cyclically loaded at $10 \mathrm{~Hz}$, with the applied stress range, $\Delta \sigma_{a p p}$, as $13.96 \mathrm{MPa}$.

\section{Results}

To assess the effectiveness of fitting parameters to the full field of data, the results are compared to those obtained from fitting cardioids to individual isopachics. In this case a simple (binary-coded) Genetic Algorithm (GA) was used as described in [5]. Level curves were extracted from the fullfield data and the GA was used to fit the parametric form in Eq. 3 (the cardioid) to the curves. The procedure is simply an evolutionary optimisation procedure which minimises an error measure between the measured data and the parametric model. Four parameters are estimated: the crack tip location (in Cartesian coordinates, so $x$ and $y$ position), the rotation of the cardioid from the horizontal, $\gamma$, and a scale factor, $P_{1}$. The parameters are related to Eq. 3 via

$$
P_{1}=\frac{K_{I}^{2}+K_{I I}^{2}}{\pi}\left(\frac{K T}{\Delta T}\right)^{2}=\frac{r_{\sigma}}{2} \quad \text { and } \quad \gamma=2 \varphi_{0}-\beta
$$

where $r_{c}$ is the maximum distance on the curve from the cusp. 


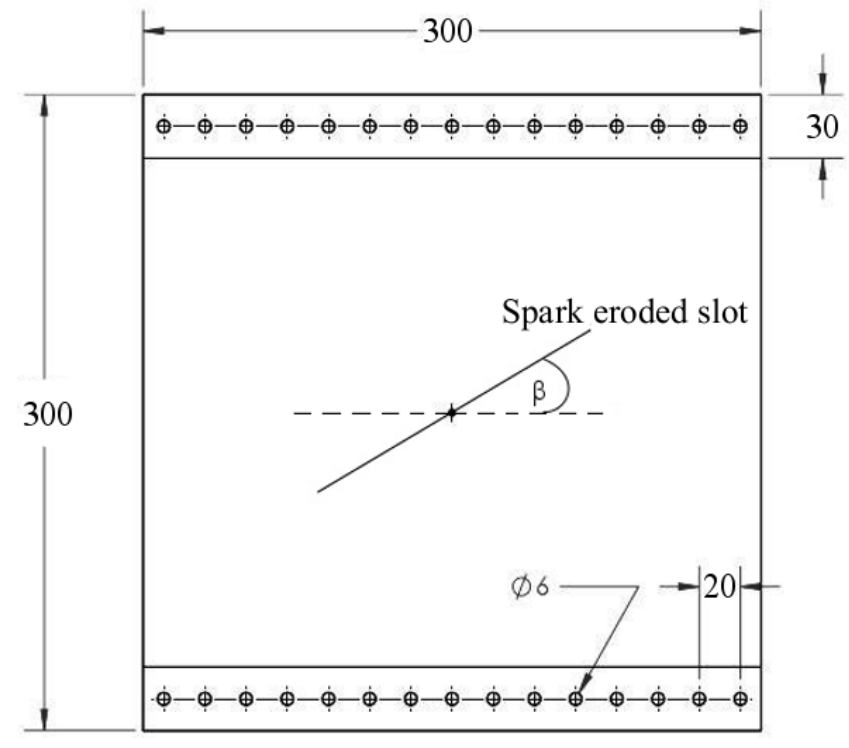

Figure 2: Schematic of the specimen

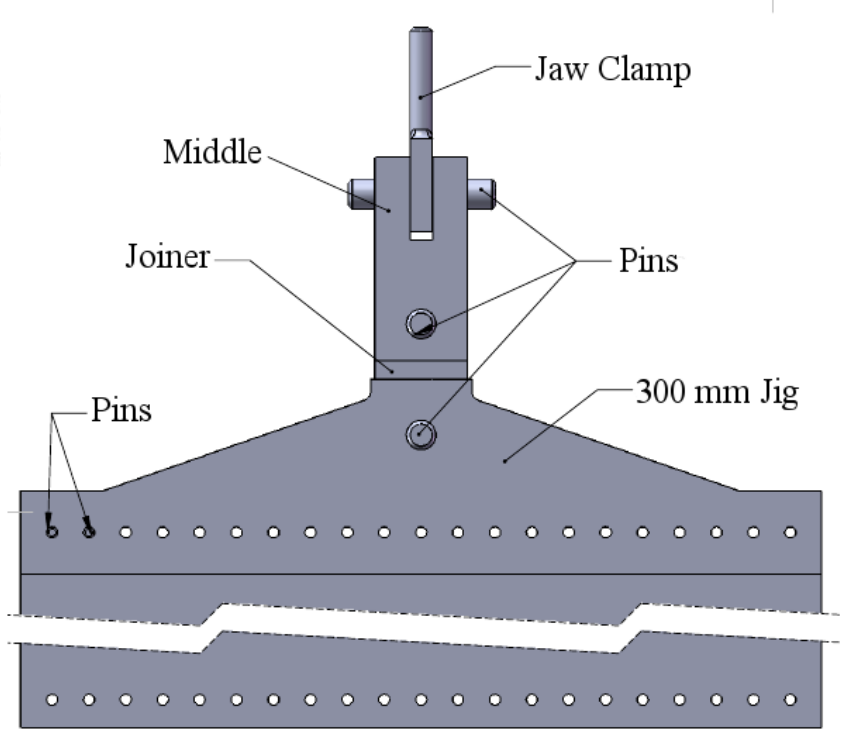

Figure 3: The loading jig

From the thermoelastic data, 10 isopachics or level curves were extracted and analysed as shown in Fig. 4; the corresponding extracted parameters are given in Table 1. The thermoelastic constant used in Eq. 5 was taken as $9.2 \times 10^{-6} \mathrm{MPa}^{-1}$. The parameters $P_{1}$ and $\gamma$ are the parameters given in Eq. 5 and the SIFs are calculated from these by simultaneously solving Eq. 5 with $\varphi_{0}=\tan ^{-1}\left(K_{I I} /\right.$ $\left.K_{I}\right)$. The difference between the calculated cusp position and the crack tip, $d r_{c}$, is also given.

Table 1: Results from curve fitting to individual extracted isopachics

\begin{tabular}{cccccccc}
\hline Set & $x[\mathrm{~mm}]$ & $y[\mathrm{~mm}]$ & $r_{c} / 2[\mathrm{~mm}]$ & $d r_{c} / 2[\mathrm{~mm}]$ & $\gamma[\mathrm{rad}]$ & $k_{I}$ & $k_{I I}$ \\
\hline 12 & 11.75 & 30.33 & 11.70 & 6.41 & 0.393 & 0.79 & 0.53 \\
13 & 10.70 & 27.73 & 8.71 & 3.65 & 0.356 & 0.75 & 0.48 \\
14 & 11.26 & 28.65 & 7.01 & 4.72 & 0.669 & 0.64 & 0.57 \\
15 & 9.99 & 26.25 & 5.78 & 2.04 & 0.332 & 0.70 & 0.44 \\
16 & 9.92 & 26.25 & 4.97 & 1.99 & 0.381 & 0.68 & 0.45 \\
17 & 9.85 & 26.32 & 4.35 & 2.01 & 0.436 & 0.67 & 0.47 \\
18 & 9.57 & 26.11 & 3.81 & 1.67 & 0.405 & 0.66 & 0.45 \\
19 & 9.64 & 26.32 & 3.40 & 1.89 & 0.504 & 0.64 & 0.48 \\
20 & 9.26 & 26.04 & 3.06 & 1.46 & 0.424 & 0.66 & 0.45 \\
21 & 9.85 & 26.67 & 2.86 & 2.29 & 0.786 & 0.57 & 0.57 \\
\hline
\end{tabular}

The actual position of the crack-tip is at $x=8.64 \mathrm{~mm}$ and $y=24.72 \mathrm{~mm}$. None of the cardioids accurately locate the crack-tip. As $r_{c}$ increases, the accuracy deteriorates; inspection of the data in Table 1 shows that sets 12, 13 and 14 furthest from the crack-tip provide the worst values. The theoretical SIF values for a crack at angle of $45^{\circ}$ are $k_{I}=0.51$ and $k_{I I}=0.51$ [4]. The results clearly show a trend converging on the theoretical values as the data approaches the crack-tip. However, even at $r_{c}$ values of much less than the slot length the values do not correspond to those given by the theory. This is because of the omission of the higher-order terms, the cardioid is not the appropriate parametric form for the level curves as the distance from the crack-tip increases. . Unfortunately, if the higher-order terms are included in the analysis, following Eq.4, the level curves (isopachics) do not have a simple parametric closed-form that makes them amenable to an analysis of the previous type. The alternative, proposed here, is to use the entire data set and use the parametric form in Eq. 4 as the basis for curve or model-fitting. This will have the added advantage of using all of the data available in the field. The parameter set for estimation now comprises 7 parameters: the $x$ and $y$ locations of the crack tip and the 5 parameters of the form in Eq. 4 Because of the enlarged 
parameter set, it is more convenient to use a real-coded evolutionary optimisation scheme so the differential evolution approach has been adopted as described in previous work [6]. As before, the objective function for the optimisation is chosen as the mean-square deviation between the model and the data.

Fig. 5 shows the experimental data with the prediction from the DE curve fit overlaid and the slot position is shown. It can be seen that the DE is providing an excellent fit to the experimental data. Table 2 gives the values of the parameters found by the DE. Here the crack-tip location is much more accurate, with the $x$-value in error by less than $1 \mathrm{~mm}$ and the $y$-value precisely located; this is a further indication that the DE curve fit is performing well.

The results presented here are from a preliminary study based on the full-field optimisation procedure; further refinement of the approach will be based on multiple runs of the DE algorithm as reported in previous work [6]. The finished methodology will allow estimation of confidence limits on the base parameters of the model and this will allow confidence intervals on the derived SIFs. Because of some uncertainty as to the appropriate form of the Williams expansion to use in the parametric model (it was reported in [9] that the basic reference [2] may contain errors), the SIF results for this data set are not presented here. What is clear from this study, however, is that the model form does allow a good reconstruction of the measured data as shown in Fig. 5. Setting aside the translation of the curve-fit parameters to SIFs; the results for the T-stress (as the uncontroversial constant term in the expansion) can be compared to Zanganeh et al [10] where the same form of the Williams expansion was used. The most similar case studied to the work presented here was biaxial loading on a cruciform with a centrally located angled slot of $\beta$ equivalent to $26^{\circ}$. The Williams expansion up to $\mathrm{O}\left(r^{1 / 2}\right)$ (i.e. as Eq. (3)) gave a T-stress value of around $20 \mathrm{MPa}$ which was about $50 \%$ of the applied stress. The current work shows the T-stress is about $20 \%$ of the applied stress. It should be noted that the two loading cases are not identical, as the crack angle is different, and therefore the T-stress will be different. However it is encouraging that the quantities are of the same order. Validation of the T-stress calculation will be carried out in future work. However, it is reported in [10] that an expansion to $\mathrm{O}(r)$ provided a much better correlation with finite element analysis.

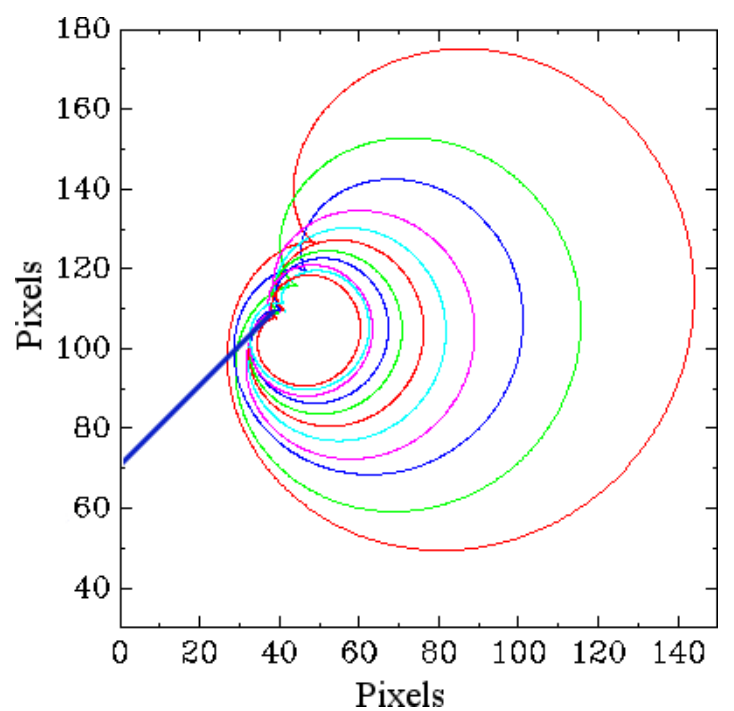

Figure 4: All of the generated curves from the first-order GA analysis.

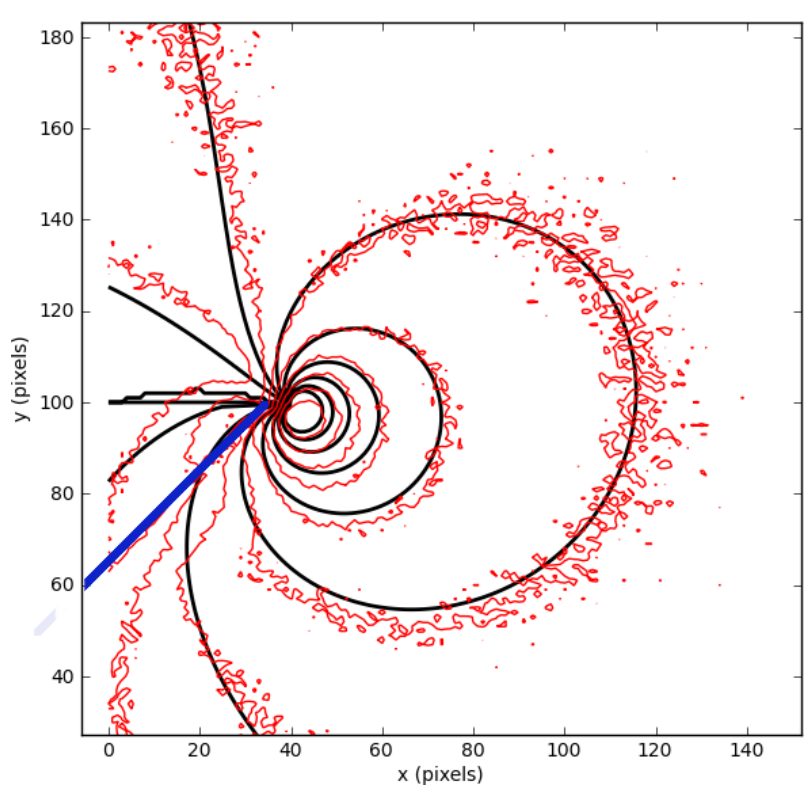

Figure 5: Experimental data with DE results over laid.

Table 2: Parameters from the DE curve fit

$\begin{array}{ccccccc}x[\mathrm{~mm}] & y[\mathrm{~mm}] & F_{1}\left[\mathrm{MPa} \mathrm{m}^{0.5}\right] & \varphi_{1}[\mathrm{rad}] & T_{s}[\mathrm{MPa}] & F_{2}\left[\mathrm{MPa} \mathrm{m}^{-0.5}\right] & \varphi_{2}[\mathrm{rad}] \\ 9.63 & 24.72 & 86.08 & -0.217 & 1.83 & 0.62 & 0.847\end{array}$


As discussed above, the relationship between the full-field parametric model and the physically significant SIF parameters will be clarified before the parameters of Table 2 are used to infer the physical constants. An issue which also requires further investigation is the effect of, the plate used to collect the data not containing a real crack, but containing a slot. Analysis of actual cracks will be the object of a future paper.

\section{Conclusions}

Previous work by the authors reached a point where it was not possible to understand the discrepancies between theoretical SIF values and those inferred from the curve-fitting procedure based on an assumed cardioid form for individual isopachics. The evidence available suggested that the discrepancies were likely arising from the fact that the cardioid form was only appropriate to the lowest-order (singular) terms in the stress field expansion. In order to accommodate higher-order terms, it was necessary to adopt a different parametric modelling approach, one based on full-field data. The preliminary study presented here shows that an evolutionary optimisation approach based on an extended parametric form for the stress field is able to provide an excellent fit to measured data. A further important conclusion also follows from this work. In the previous studies, the fitting of individual isopachics did not allow an explanation as to why the fitted curves appeared to rotate with increasing distance from the crack-tip. The parametric form adopted here shows clearly that the rotation is a consequence of the higher-order terms in the stress field expansion and that the rotation can be effectively captured by the model-fitting procedure.

\section{References}

[1] Ewalds, H.L. and Wanhill, R.J. Fracture Mechanics. Arnold, London, 1991

[2] Williams, M.L. On the stress distribution at the base of a stationary crack. Journal Applied Mechanics, 24 (1957)

[3] Dulieu-Smith, J.M. and Stanley, P. Progress in the thermoelastic evaluation of mixed-mode stress intensity factors. In Proc. SEM Spring Conf. On Expt. Mech. (Dearborn 1993), 617629.

[4] Dulieu-Barton, J.M., Fulton, M.C. and Stanley, P. The analysis of thermoelastic isopachic data from crack-tip stress fields. Fatigue Fract. Engng. Mater. Struct., 23 (1999), 301-313.

[5] Dulieu-Barton, J.M. and Worden, K. Genetic identification of crack-tip parameters using thermoelastic parameters. Meas. Sci. Tech., 14 (2002), 176-183.

[6] Dulieu-Barton, J.M. and Worden, K., Identification of crack-tip parameters using thermoelastic isopachics and differential evolution. Key Engineering Materials, 245-246 (2003), 77-86

[7] Hebb, R.I, Dulieu-Barton, J.M., Worden, K. and Tatum, P. Curve fitting of mixed-mode isopachics. 7th International Conference on Modern Practice in Stress and Vibration Analysis, Journal of Physics, 8-10 September, 2009

[8] Dulieu-Barton, J.M. and Stanley, P. Development and applications of thermoelastic stress analysis. Journal of Strain Analysis for Engineering Design, 33 (1998), 93-104

[9] Berto, F. And Lazzarin, P. On higher order terms in the crack tip stress field. Int. J. Fract., 161 (2010), 221-226

[10] Zanganeh, M., Tomlinson, R.A. and Yates, J.R., T-stress determination using thermoelastic stress analysis. Journal Strain Analysis, 43 (2008), 529-537 